\title{
Design and implementation of 4-bit binary weighted current steering DAC
}

\author{
Jayeshkumar J. Patel, Amisha P. Naik
}

Department of Electronics and Communication Engineering, Institute of Technology, Nirma University Ahmedabad, India

\begin{tabular}{l} 
Article Info \\
\hline Article history: \\
Received Mar 20, 2019 \\
Revised May 9, 2020 \\
Accepted May 27, 2020 \\
\hline Keywords: \\
CMOS current-steering DAC \\
DAC \\
DNL \\
INL \\
Transmission gate
\end{tabular}

\begin{abstract}
A compact current-mode Digital-to-Analog converter (DAC) suitable for biomedical application is repesented in this paper. The designed DAC is binary weighted in $180 \mathrm{~nm}$ CMOS technology with $1.8 \mathrm{~V}$ supply voltage. In this implementation, authors have focused on calculaton of Non linearity error say INL and DNL for 4-bit DAC having various type of switches: NMOS, PMOS and transmission gate. The implemented DAC uses lower area and power compared to unary architecture due to absence of digital decoders. The desired value of Integrated non linearity (INL) and Differential non linearity (DNL) for DAC for are within a range of +0.5LSB. Result obtained in this works for INL and DNL for the case DAC using transmission gate is $\pm 0.34 \mathrm{LSB}$ and \pm 0.38 LSB respectively with $22 \mathrm{~mW}$ power dissipation.
\end{abstract}

Copyright $@ 2020$ Institute of Advanced Engineering and Science. All rights reserved.

\section{Corresponding Author:}

Jayeshkumar J. Patel,

Department of Electronics and Communication Engineering,

Institute of Technology, Nirma University,

S G Highway, Ahmedabad, Gujarat, India.

Email: jayesh.patel@nirmauni.ac.in

\section{INTRODUCTION}

The Digital to Analog converter (DAC) is a circuit which converts digital signal into analog one. It is widely used in digital signal processors. DACs are often used to convert finite- precision time series to a varying physical data. These are mainly used in different applications like data distribution and acquisition systems, amplifier, Electronics display [1-7].

The Current steering DACs are the more commonly used architecture because of their small size and simplicity, high resolution and high speed. Based on the binary principle, current sources are scaled. Here for $\mathrm{i}^{\text {th }}$ current source, output current is equal to the $2^{\mathrm{i} * \mathrm{I}}$, Where $\mathrm{I}=$ Least significant bit (LSB) current. For the design of DAC, various switches like NMOS, PMOS and transmission gate are explored. Characteristics of switching elements are one of the prominent factors for dynamic non linearity of DAC [8,9].

In the proposed 4-bit DAC, four binary weighted current sources are used, those are represented as: $\mathrm{I}_{0}, 2 \mathrm{I}_{0}, 4 \mathrm{I}_{0}$ and $8 \mathrm{I}_{0}$. The main advantage of this architecture is number of current cells required will be same as no. of bits. Hence, this architecture is most suitable for higher resolution implementations. The disadvantage with this architecture: it produces number of glitches (unwanted signal) on the contrary the unary architecture offers higher accuracy with greater linearity at the cost of chip area and power overhead $[10,11]$.

\section{CURRENT STEERING DAC}

DAC are vailable in form of various architectures: Decoder based DAC, Weighted R DAC, R-2R Ladder DAC, charged DAC, Cuurent steering DAC. Compared to other Architecturrs, Current steering DAC 
is faster and low power consuming. There is no need of extra buffer to drive a load. A current steering DAC uses a reference current source. The source is replicated in each branch of DAC. The current sources belong to the branch is switched on or off according to digital inputs. In case of binary weighed current steering DAC, Current source having the value of $2^{\mathrm{N}} *$ Io. Where Io is reference current. No. of switches are same as no. of current sources and same as N. Based on ON/OFF of current sources, total current is added and it will be the output current. Switches are MOS switches- NMOS, PMOS or transmission gate and sane are controlled directly by digital inputs. Output current is as per input code [12-18]. N-bit DAC is represented as low shown in Figure 1.

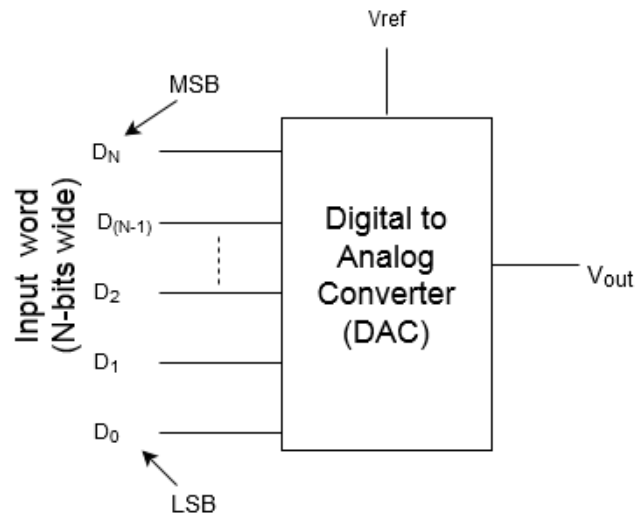

Figure 1. N-bit DAC representation

For N-bit DAC, output is expressed as follow:

$$
\begin{aligned}
& V_{\text {OUT }}=\left(D_{N-1} 2^{N-1}+\ldots+D_{0} 2^{0}\right) \frac{V_{R E F}}{2^{N}} \\
& V_{\text {OUT } \max }=\frac{2^{N-1}}{2^{N}} \cdot V_{R E F}
\end{aligned}
$$

A 4-bit binary weighted current steering DAC is designed and implemented with various switching approaches suitable for biomedical application. Though this architecture occupies lesser digital area and power, but suffers from glitches specifically when have more numbers of transitions in input. The authors have calculated INL, DNL of 4-bit Binary Current Steering DAC having various type of switches: NMOS, PMOS and transmission gate [9, 12]. DAC are evaluated based on the various parameters like Resolution, poer concumption, setteling time, dynamic range, non-linearity error (INLand DNL). In this paper, focus is given on INL and DNL. Differential nonlinearity (acronym DNL) represents a deviation of actual step size with reference to ideal step size, where step size is a difference of analog outputs for adjacent input values $[6,10]$. Mathematically DNL for DAC is represented as follow:

$$
\operatorname{DNL}(i)=\frac{V_{\text {out }}(i+1)-V_{\text {out }}(i)}{\text { ideal LSB step width }}-1
$$

Integral nonlinearity (acronym INL) represents a deviation of actual analog output of DAC with reference to expected ideal value for given digital input value. It is also expressed in terms of DNL as follow [6, 10].

$$
\operatorname{INL}(n)=\sum_{k=0}^{n} D N L(k)
$$

The characteristics of Switch play an important role for high speed, low power and high-resolution DAC. Which decides the Non linearity say DNL and INL of DAC. There are other architectures in implementation say unary current steering DAC. Said architecture is complex in terms of number of current sources. Here each current source has the same value of Io (reference current) but number of current sources are $\left(2^{\mathrm{N}}-1\right)$ and same no. of switches as well. It offers advantage in form of less glitches but required more 
area. It is also required to have additional hardware to convert binary code into thermometer code [19-22].

Looking to implementation, area efficiency and free from additional hardware for binary to thermometer code conversion, Binary current steering DAC structure is the simplest one. $\mathrm{N}$ bit configuration which needs only $\mathrm{N}$ current sources. They are straight-forwardly worked by the linear binary input codes. However, due to the inadequate synchronization of the switches and dynamic behavior of the circuit, large glitches in form of impulses are observed at the output terminal. This problem is addressed using better switch. This structure also offers a merit in form of less no. of transistors as well. The 4-bit binary weighted current steering DAC is as shown in Figure 2 [17, 20, 23-25].

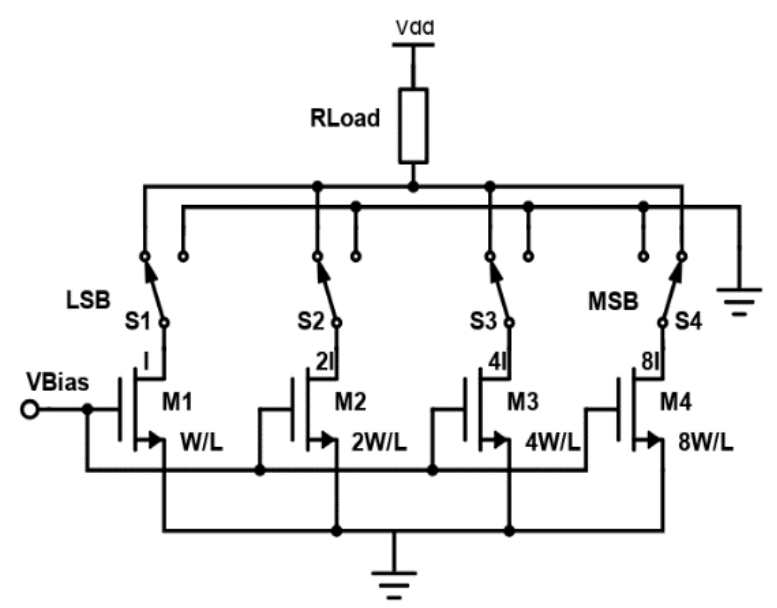

Figure 2. 4-bit binary weighted current steering DAC

The present work is focused to design and analyse the effect of various types of switches on non linearity eroor say DNL and INL. Based on understanding from the literature survey, three types of switches say NMOS, PMOS and transmission gate have been tried and simulation of each one has been carried out. For the proposed design and simulation, cadence tool is used with $180 \mathrm{~nm}$ CMOS technology. The proposed current steering DAC offers desired INL and DNL with rated power consumption.

\section{VARIOUS SWITCHING APPROACHES}

A main source of nonlinearity originates because of glitches in the current cell. More no. of transitions results more no. of changes the states of switches say on to off or vice versa. In case of 4-bit binary weighted DAC, when input changes from 0011 to 0100 , big glitch is observed because of 3 transitions. Similarly, when input changes from 0111 to 1000 , even big glitch will be there as there are 4 transitions. In case of unary weighted DAC, there is only 1-bit transition so there is no glitch but it needs more no. of current sources; for 4-bit unary current steering DAC, 15 current sources of having same value are required. Thermometer code is used to control the switches. Additional hardware is required to convert binary code into thermometer codes [26-29].

Characteristics of switch also play an important role. There are various options for the same: NMOS, PMOS, Transmission gate. NMOS and PMOS are used as a single device and controlled by sinlge input. While transmission gate is parallel combination of NMOS and PMOS and complementary control inputs are desired in this case [22].

\section{SIMULATION RESULTS AND DISCUSSIONS}

4-bit binary weighted current steering DAC have been implemented using cadence virtuoso in CMOS $180 \mathrm{~nm}$ technology. This converter developed and simulated in a $180 \mathrm{~nm}$ CMOS technology with supply voltage of $1.8 \mathrm{~V}$. Based on three different type of switches, three possible combinations have been simulated. DNL and INL were calculated for all three DACs. It has been observed that the DNL and INL in case of DAC having Transmission gate are as \pm 0.42 LSB and $\pm 0.4 \mathrm{LSB}$, respectively. With the operating frequency of $200 \mathrm{Mhz}$, simulated power consumption was $22 \mathrm{~mW}$. 
Authors represented and compared three architectures and their outputs in form of currents. Figure 3 to Figure 8 shows the simulated results and output of proposed segmented DAC using various kinds of switches say NMOS, PMOS and transmission gate. Each architecture having two parts: one is current mirror and second is switching elements, current mirror part is common in all three architectures.

In case of NMOS switch-based architecture, big glitches have observed and same results poor non linearity. Glitches are available when there are a greater number of transitions in digital inputs e.g when input change from 0111 to 1000 , prominent glitch is there. The step size should be equal but it is observed that even in some cases of input changes, it is reduced rather than to be increased. Same will have adverse impact on non-linearity error in terms of INL as well as DNL. Here in case of PMOS switch, same kind of observations are there as observed in case of NMOS switches. As digital input increase, output should increase. It is not always observed in case of NMOS and PMOS kinds of switches.

Transmission gate is one good option as a switch. Architecture having transmission gate offers a big advantage in form of reduction of glitches as well as continuous rise of current as desired which makes lesser value of INL and DNL. Graphs for INL and DNL of proposed current steering DAC using transmission gate switches are represented in Figure 9 and Figure 10 respectively. The simulated result of binary weighted DAC using transmission gate as a switch are as shown in Table 1.

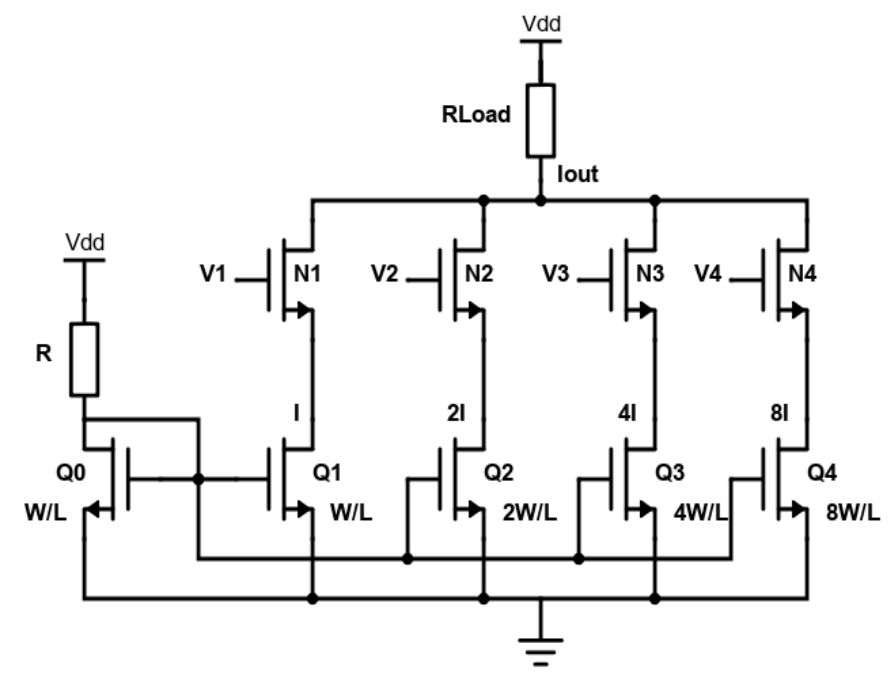

Figure 3. Architecture of binary weighted DAC with NMOS switches

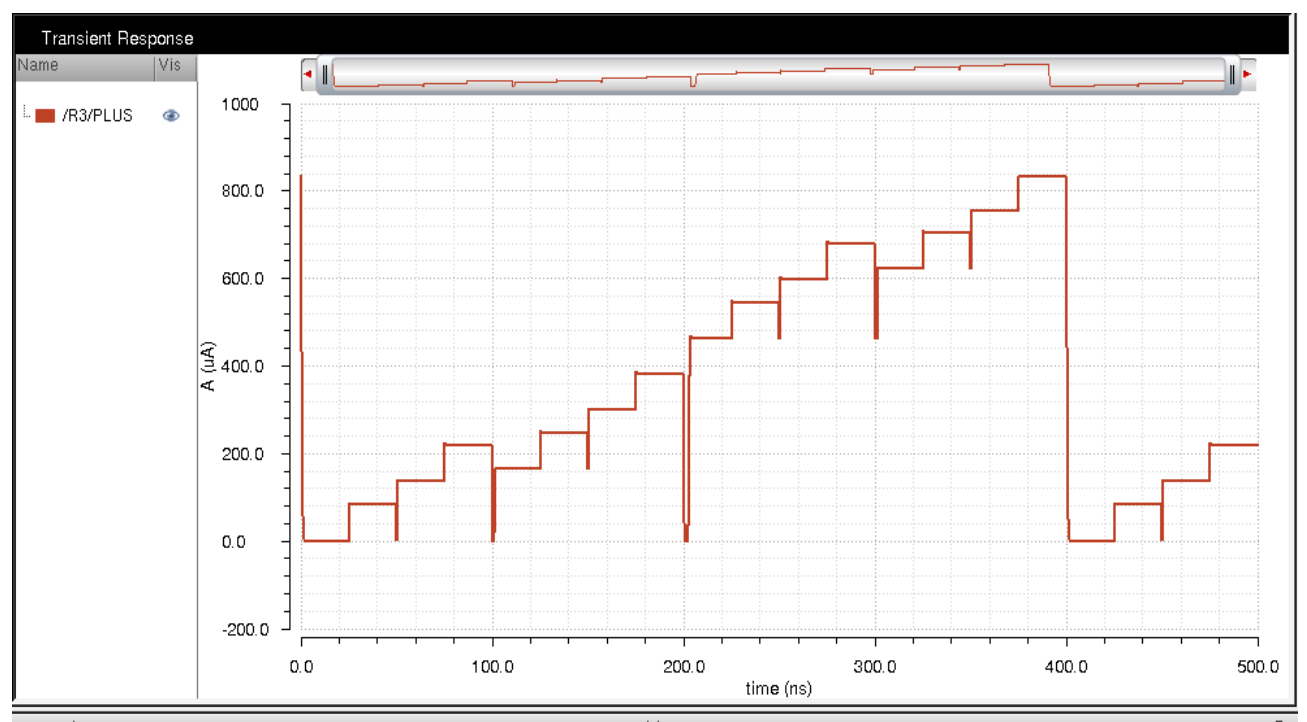

Figure 4. Current output of binary weighted DAC with NMOS switches 


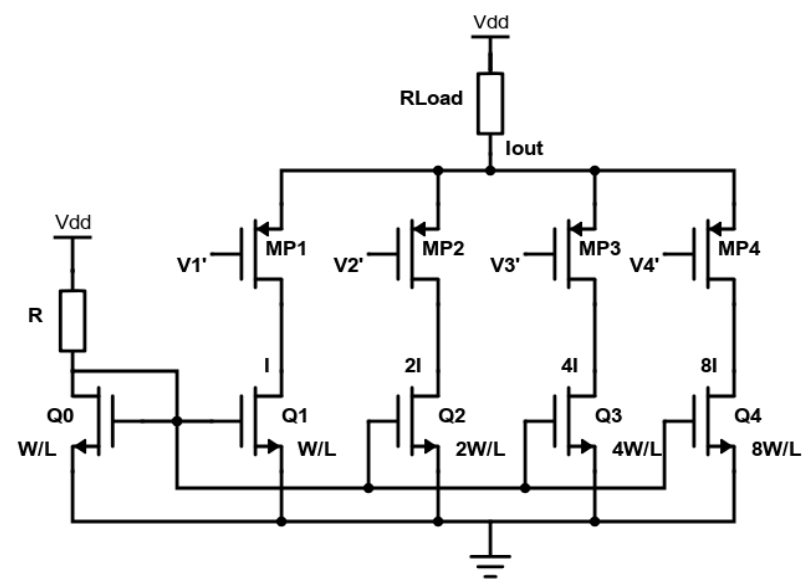

Figure 5. Architecture of binary weighted DAC with PMOS switches

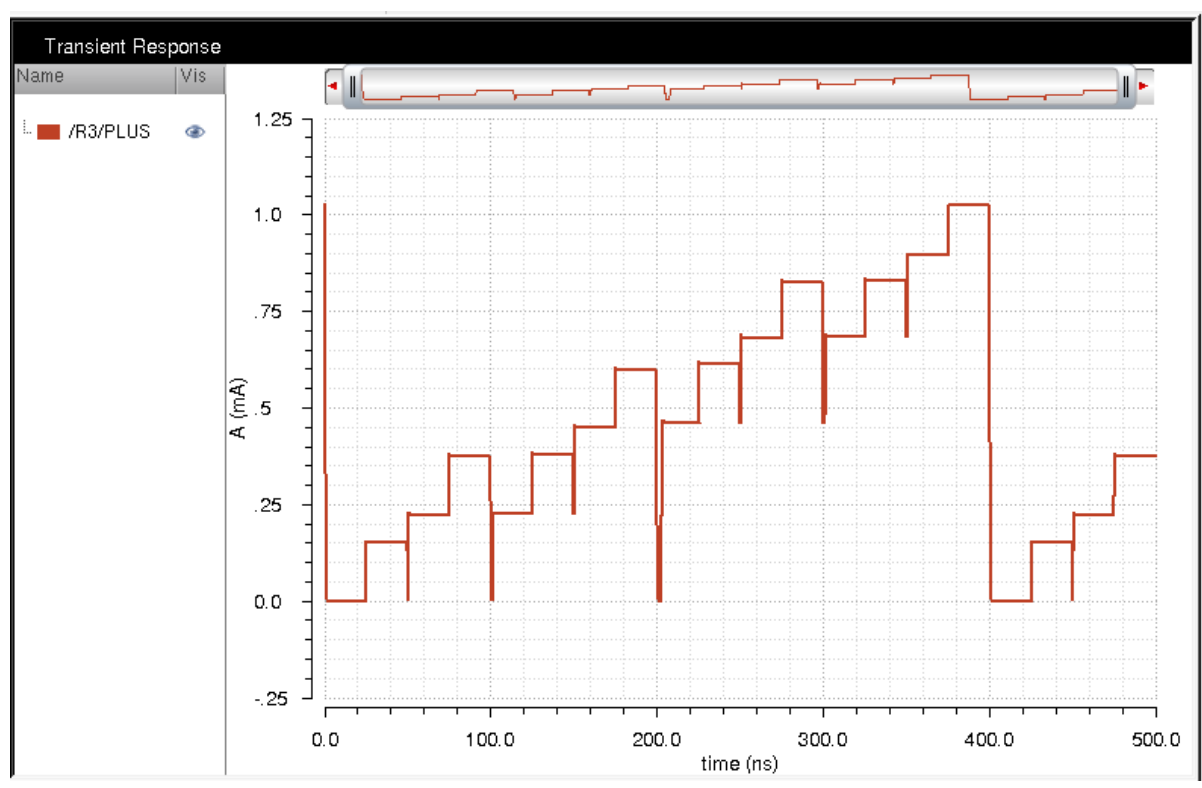

Figure 6. Current output of binary weighted DAC with PMOS switches

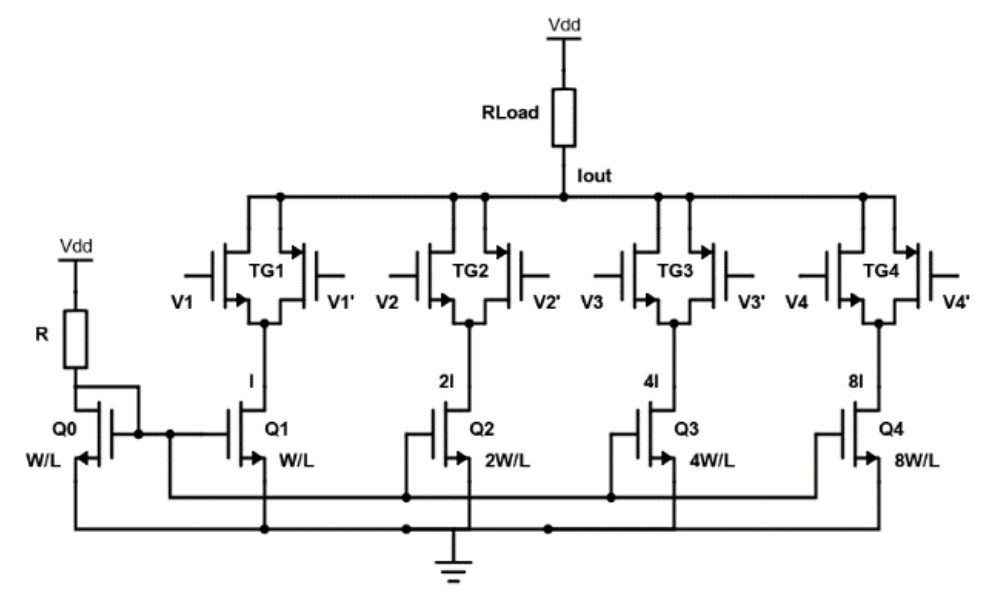

Figure 7. Architecture of binary weighted DAC with transmission gate switches 


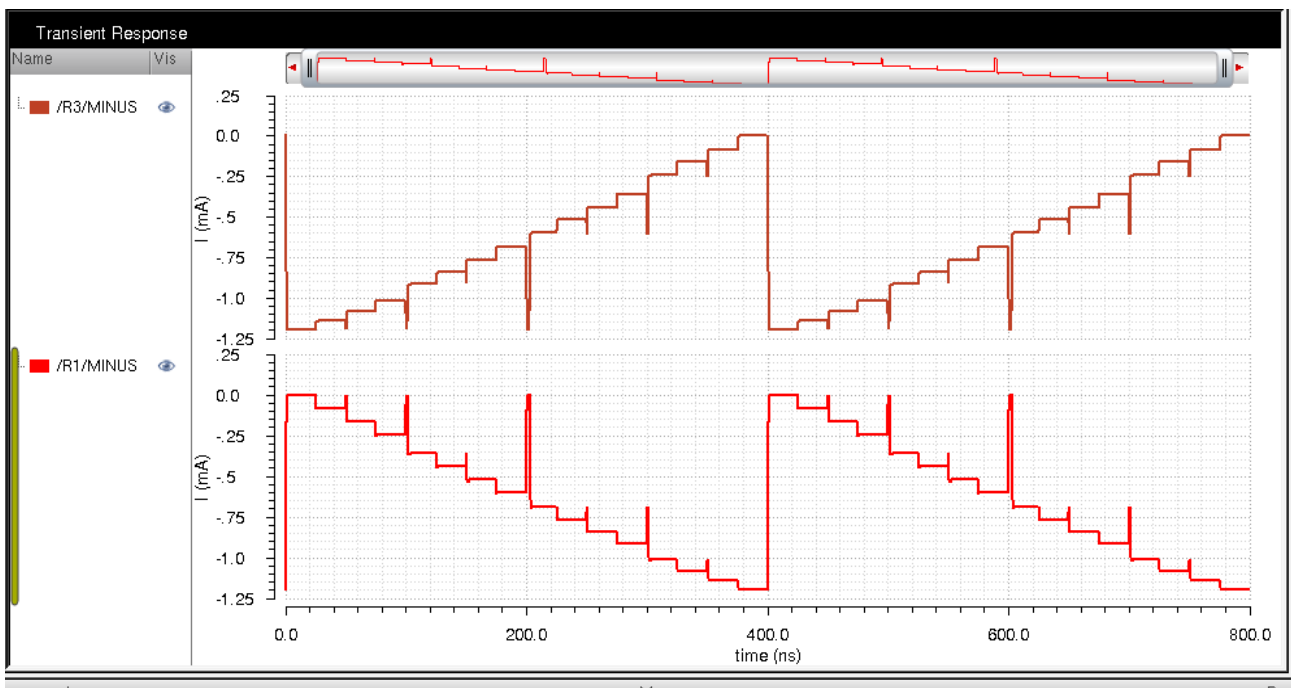

Figure 8. Current output of binary weighted DAC with transmission gate switches

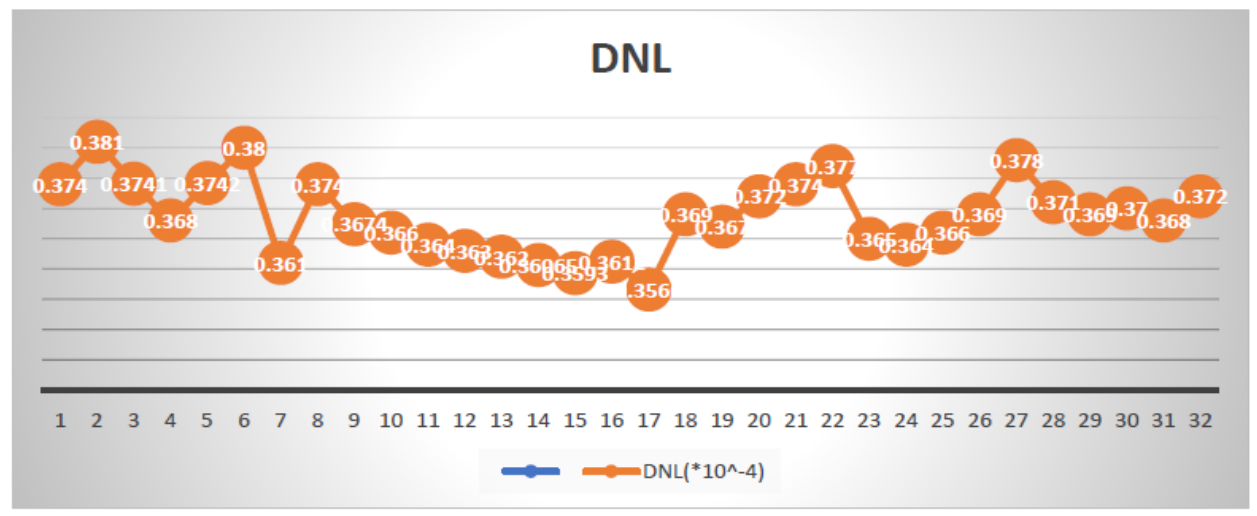

Figure 9. DNL graph of 4 bit binary weighted DAC having transmission gate

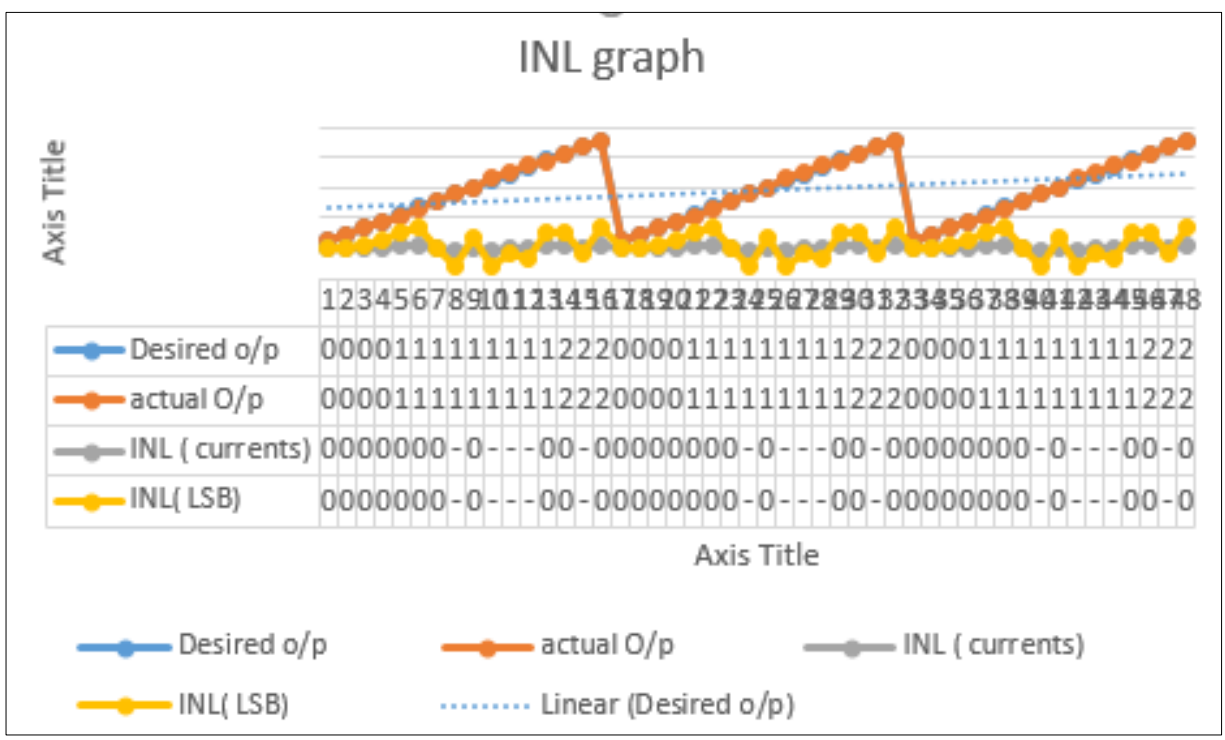

Figure 10. INL graph of 4 bit binary weighted DAC having transmission gate 
Table 1. Simulated result of DAC

\begin{tabular}{cccc}
\hline Parameters & Value & Parameters & Value \\
\hline Technology & 180 & INL (Max) & $0.34 \mathrm{LSB}$ \\
Resolution & 4-bit & DNL (Max) & $0.38 \mathrm{LSB}$ \\
Approach & Binary weighted & Power (Max) & $22 \mathrm{~mW}$ \\
Supply voltage & $1.8 \mathrm{~V}$ & Frequency & $200 \mathrm{Mhz}$ \\
\hline
\end{tabular}

\section{CONCLUSION}

A binary weighted 4 bit current-mode digital to Analog converter (DAC) useful in the field of biomedical application designed and simulated using 180nm CMOS Process.In this implementation the authors have calculated INL and DNL of DAC having NMOS, PMOS and transmission gate as a switch. It is desired to have INL and DNL in the range of $\pm 0.5 \mathrm{LSB}$. Based on comparison. It has been observed that Digital to Analog convertor with transmission gate as a switch, DNL and INL are 0.38 LSB and 0.34 LSB respectively. Power consumption is observed as $22 \mathrm{~mW}$.

\section{REFERENCES}

[1] C. Seok, H. Kim, S. Im, H. Song, K. Lim, Y.-S. Goo, K.-i. Koo, D.-i. Cho and H. Ko, "A 16-channel neural stimulator IC with DAC sharing scheme for artificial retinal prostheses," Journal Of Semiconductor Technology And Science, vol. 14, pp. 658-665, 2014.

[2] S. Sarkar and S. Banerjee, "An 8-bit low power DAC with re-used distributed binary cells architecture for reconfigurable transmitters," Microelectronics Journal, vol. 45, pp. 666-677, 2014.

[3] S. Sarkar and S. Banerjee, "A 10-bit 500 MSPS Segmented DAC with Optimized Current Sources to Avoid Mismatch Effect," IEEE Computer Society Annual Symposium on VLSI, Montpellier, pp. 172-177, 2015.

[4] B. Razavi, "Design of analog CMOS integrated circuits," Tata McGraw-Hill Education, NY, 2002.

[5] M. T. Rahman and T. Lehmann, "A self-calibrated cryogenic current cell for $4.2 \mathrm{~K}$ current steering D/A converters," IEEE Transactions on Circuits and Systems II: Express Briefs, vol. 64, pp. 1152-1156, 2016.

[6] D. Przyborowski and M. Idzik, "A 10-bit low-power small-area high-swing CMOS DAC," IEEE Transactions on Nuclear Science, vol. 57, pp. 292-299, 2010.

[7] G. Park and M. Song, "A CMOS current-steering D/A converter with full-swing output voltage and a quaternary driver," IEEE Transactions on Circuits and Systems II: Express Briefs, vol. 62, pp. 441-445, 2014.

[8] N. Pal, P. Nandi, R. Biswas and A. G. Katakwar, "Placement-based nonlinearity reduction technique for differential current-steering DAC," IEEE Transactions on Very Large Scale Integration (VLSI) Systems, vol. 24, no. 1, pp. 233-242, 2015.

[9] I. Mukhopadhyay, M. Y. Mukadam, R. Narayanan, F. O'Mahony and A. B. Apsel, "Dual-calibration technique for improving static linearity of thermometer DACs for I/O," IEEE Transactions on Very Large Scale Integration (VLSI) Systems, vol. 24, no. 3, pp. 1050-1058, 2015.

[10] S. N. Mohyar and H. Kobayashi, "Digital calibration algorithm for half-unary current-steering DAC for linearity improvement," 2014 International SoC Design Conference (ISOCC), Jeju, pp. 60-61, 2014.

[11] P. Mathurkar and M. Mali, "Segmented 8-bit current-steering digital to analog converter," 2015 International Conference on Pervasive Computing (ICPC), Pune, pp. 1-4, 2015.

[12] R. Liu and L. Pileggi, "Low-overhead self-healing methodology for current matching in current-steering DAC," IEEE Transactions on Circuits and Systems II: Express Briefs, vol. 62, no. 7, pp. 651-655, 2015.

[13] R. J. Baker, "CMOS: circuit design, layout, and simulation," John Wiley \& Sons, 2019.

[14] P. E. Allen and D. R. Holberg, "CMOS analog circuit design," Elsevier, 2011.

[15] M. S. Yenuchenko, "Alternative structures of a segmented current-steering DAC," 2018 International Symposium on Consumer Technologies (ISCT), St. Petersburg, pp. 14-17, 2018.

[16] D.-L. Shen, Y.-C. Lai and T.-C. Lee, "A 10-bit binary-weighted DAC with digital background LMS calibration," 2007 IEEE Asian Solid-State Circuits Conference, Jeju, pp. 352-355, 2007.

[17] H.-C. Seol, S.-K. Hong and O.-K. Kwon, "An area-efficient high-resolution resistor-string DAC with reverse ordering scheme for active matrix flat-panel display data driver ICs," Journal of Display Technology, vol. 12, no. 8, pp. 828-834, 2016.

[18] D. Seo, "A heterogeneous 16-bit DAC using a replica compensation," IEEE Transactions on Circuits and Systems I: Regular Papers, vol. 55, no. 6, pp. 1455-1463, 2008.

[19] R. Rubino, P. S. Crovetti and O. Aiello, "Design of Relaxation Digital-to-Analog Converters for Internet of Things Applications in 40nm CMOS," 2019 IEEE Asia Pacific Conference on Circuits and Systems (APCCAS), Bangkok, Thailand, pp. 13-16, 2019.

[20] C.-W. Lu, C.-M. Hsiao and P.-Y. Yin, "A 10-b two-stage DAC with an area-efficient multiple-output voltage selector and a linearity-enhanced DAC-embedded op-amp for LCD column driver ICs," IEEE journal of solid-state circuits, vol. 48, no. 6, pp. 1475-1486, 2013.

[21] W.-T. Lin and T.-H. Kuo, "A compact dynamic-performance-improved current-steering DAC with random rotation-based binary-weighted selection," IEEE Journal of Solid-State Circuits, vol. 47, no. 2, pp. 444-453, 2011. 
[22] D.-K. Jung, Y.-H. Jung, T. Yoo, D.-H. Yoon, B.-Y. Jung, T. T.-H. Kim and K.-H. Baek, "A 12-bit multi-channel RR DAC using a shared resistor string scheme for area-efficient display source driver," IEEE Transactions on Circuits and Systems I: Regular Papers, vol. 65, no. 11, pp. 3688-3697, 2018.

[23] X. Huo, W. Bai, H.-M. Lam, C. Liao, M. Zhang, S. Zhang and H. Jiao, "A Compact Low-Voltage Segmented D/A Converter with Adjustable Gamma Coefficient for AMOLED Displays," 2019 IEEE International Symposium on Circuits and Systems (ISCAS), Sapporo, Japan, pp. 1-5, 2019.

[24] J. Dalecki, R. Dlugosz, T. Talaska and G. Fischer, "A Low Power, Low Chip Area, Two-stage Current-mode DAC Implemented in CMOS $130 \mathrm{~nm}$ Technology," 2019 MIXDES - 26th International Conference, "Mixed Design of Integrated Circuits and Systems," Rzeszów, Poland, pp. 151-156, 2019.

[25] H. Cruz, T.-C. Yeh, H.-Y. Huang, S.-Y. Lee and C.-H. Luo, "DAC for positron emission tomography front-end," 2014 IEEE International Symposium on Bioelectronics and Bioinformatics (IEEE ISBB 2014), Chung Li, pp. 1-4, 2014.

[26] H. Cruz, H.-Y. Huang, C.-H. Luo, L.-Y. Chiou and S.-Y. Lee, "A novel clock-pulse-width calibration technique for charge redistribution DACs," 2017 IEEE International Symposium on Circuits and Systems (ISCAS), Baltimore, $M D$, pp. 1-4, 2017.

[27] F.-T. Chou, Z.-Y. Chen and C.-C. Hung, "A 10-bit 250MS/s low-glitch binary-weighted digital-to-analog converter," in 2014 27th IEEE International System-on-Chip Conference (SOCC), Las Vegas, NV, 2014, pp. 231-235, 2014.

[28] O. Aiello, P. Crovetti and M. Alioto, "Standard Cell-Based Ultra-Compact DACs in 40-nm CMOS," IEEE Access, vol. 7, pp. 126479-126488, 2019.

[29] Jayesh Patel, Amisha Naik. "A Low Voltage High Speed Segmented Current Steering DAC for Neural Stimulation Application," International Journal of Recent Technology and Engineering, vol. 8, no. 5, pp. 4270-4274, 2020.

\section{BIOGRAPHIES OF AUTHORS}

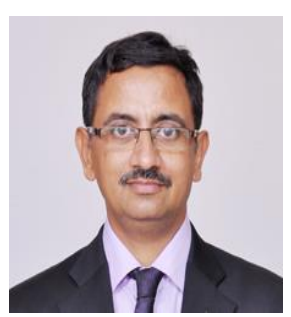

Jayeshkumar J. Patel is pursuing his Ph. d from Nirma University, Ahmedabad in the field of mixed signal circuit design. He obtained his M. Tech in EC (VLSI Design) from Institute of Technology, Nirma University, Ahmedabad in 2007. He did his B.E from BVM College of Engineering, Vallabh Vidyanagar. He has published many technical papers in national and international journals. He has more than 18 years of experience in teaching at UG \& PG levl. He also worked for industry for about 5 years. His research interest includes Analog and Mixed signal VLSI

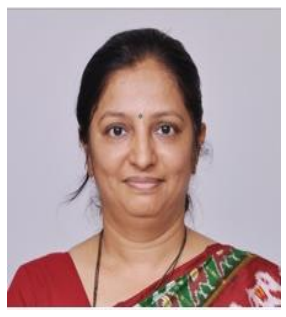

Dr. Amisha Naik is working as Associate professor at EC department, Nirma University, Ahmedabad since 2001. She teaches both at UG and PG level. She did B. E in Electronics from Regional Engineering College, Surat in the year 1997 and obtained M. Tech and Ph. D in VLSI Design area in the year 2006 and 2011 respectively from Nirma University, Ahmedabad. She has published many papers in national and international journals. She is guiding several Ph.D students in the area of analog and mixed signal circuit design. 